\title{
MULTI-SENSORIAL SYSTEM FOR THE GENERATION OF DISASSEMBLY TRAJECTORIES
}

\author{
J. POMARES, UNIVERSITY OF ALICANTE, SPAIN, jpomares@ua.es. \\ S. T. PUENTE, UNIVERSITY OF ALICANTE, SPAIN, Santiago.Puente@ua.es. \\ G. J. GARCÍA, UNIVERSITY OF ALICANTE, SPAIN, gjgg@ua.es. \\ F. TORRES, UNIVERSITY OF ALICANTE, SPAIN, Fernando.Torres@ua.es.
}

\begin{abstract}
The disassembly of products for they reuse is an important field of research. One aspect that appears when a product is been disassembled is the uncertainty in the trajectories for the removal of a component from the rest of the product. In order to reduce this lack of information, a multisensorial system is proposed. This system uses visual and force-sensor information to avoid the unknown factors in the disassembly plan and correct the trajectories generated which are based on the model of the product. This paper also describes the structure of a de-manufacturing system that can perform the desired disassembly task
\end{abstract}

KEYWORDS: disassembly, multi-sensorial system, de-manufacturing, robotics, data fusion.

\section{INTRODUCTION}

Disassembly is of great interest in current research as its aim is to achieve the optimal recovery and recycling of the components of a product. There are great benefits for both industry and the environment in recovering components from a product when its life-cycle has ended. When the components are recovered it reduces the number of new components that must be manufactured, thus reducing costs in the manufacturing process and in the amount of waste generated [6][1]. When a disassembly task is to be performed, an important issue to be taken into consideration is the difference between the expected aspects of the component and the real ones. The component to be disassembled may change during its life-cycle, due to the way it has been used, and the environment in which it has been applied. Some sensorial system is therefore needed to achieve the correct model of the product that is being disassembled. It may also be a vision-system [3] that allows the obtaining of information about the real product.

Using this approach, the product can be modelled correctly to be able to define the disassembly tasks to be performed [4], reaching the target component. Furthermore, when a specific component is to be separated from the rest of the product, the path to be followed for its removal must be known. Once the path has been defined, it must be followed by a robot if the disassembly tasks are to be performed automatically. When the robot is following the path, unexpected problems may arise, like an unknown contact element, the appearance of cables obstructing the trajectory, the gripper not being perfectly perpendicular to the grasping point, and so on. Therefore, some reactive system has to be used to avoid such unexpected problems.

For dealing with this problem we propose a de-manufacturing system, which uses a robot arm and a multi-sensorial system to achieve the desired disassembly of the product. The multisensorial system is also composed of a vision-system and a force-sensor that gives information when the vision-system does not give enough information to accomplish the desired task.

In the following section, the structure of the de-manufacturing system and the architecture employed is outline. Section 3 presents the multi-sensorial system used to perform the disassembly task. In Section 4 the results of this method are presented and, finally the conclusions obtained and the bibliography employed are presented. 


\section{DEMANUFACTURING SYSTEM}

In this section, the structure of the de-manufacturing system is described. Figure 1 shows the main modules of the system and the different parts that make up the system.

The database contains information about the products to be disassembled: i.e., models of the different components that make up the products, and of which there are geometric models, as well as their identifying characteristics. The multi-sensorial system can be used for the recognition and location of the products and their components [3]. The global planner fuses the information that comes from the multi-sensorial system and from the database for making the particular model of the product that is going to be disassembled at that moment. This part of the systems allows changing from a model of the family of products that is to be disassembled to a model of the specific product that is being disassembled, considering its particular characteristics. The precise model of a product represents the hierarchical relations among its components. This module also generates a geometric model of the particular product.

The sequence planner uses the hierarchical model of the product to compute a total or partial disassembly sequence of a product [9]. It computes a sequence of actions to be performed for separating the components of the product that do not allow access to the desired one.

The movement generator uses the geometric model generated by the global planner for computing the path to be followed for separating one specific component of the product from the rest of components [7][8]. When a path is computed and has to be performed by the robot, different problems may appear. These problems represent the differences between the real product and the modeled one. To solve these problems in a proper way, we propose the use of the multi-sensorial system to correct the path generated by the movement generator.

The disassembly system is composed of a PA-10 robot-arm with 7 degrees of freedom and a work table with the product to be disassembly on it.

The robot-arm has an eye-in-hand configuration to perform the visual-control. The micro-head camera used is a JAI-M536 model. Added to the eye-in-hand configuration the robot arm is complemented with a JR3 force-sensor, which allows the measurement of the interaction forces generated when a disassembly task is performed (Figure 1).
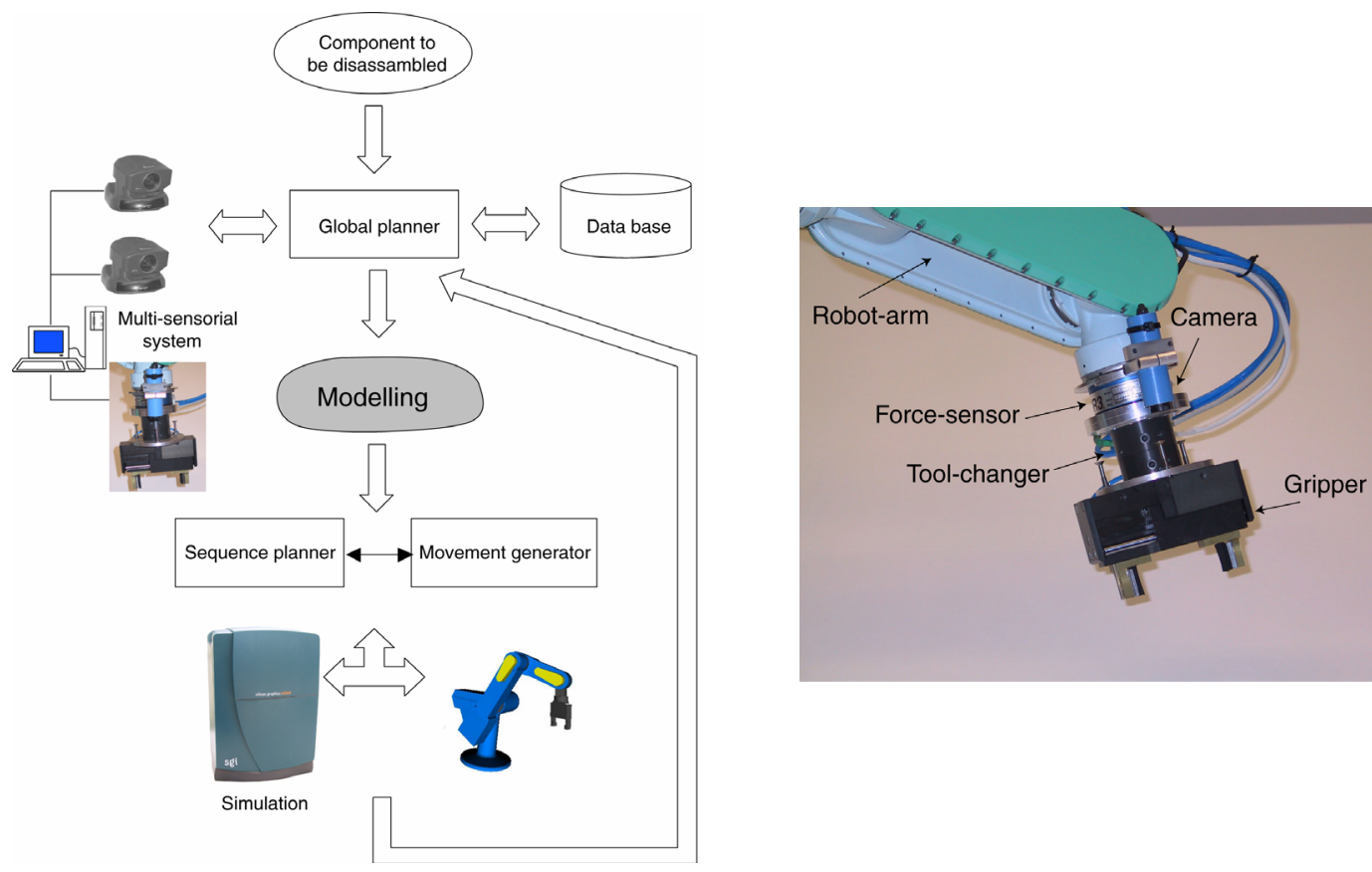

Figure 1. Structure of the automatic de-manufacturing system. 


\section{MULTISENSORIAL SYSTEM}

Employing the demanufacturing system described in Section 2, the desired trajectory in the 3D space to be tracked, is generated. By sampling this trajectory the set $\tau=\left\{{ }^{k} \boldsymbol{p}_{\mathrm{o}}^{\mathrm{X}} / \mathrm{k} \in 1 \ldots \mathrm{N}\right\}$, is obtained, in which ${ }^{\mathrm{k}} \boldsymbol{p}_{\mathrm{o}}^{\mathrm{X}}$ is the location of the object $\mathrm{O}$ with respects to the coordinate frame $\mathrm{X}$ at the instant $\mathrm{k}$ (the trajectory being specified with respect to the object $\mathrm{O}$ ). ${ }^{\mathrm{k}} \boldsymbol{p}_{\mathrm{o}}^{\mathrm{X}}$ is projected in the image at the instant $\mathrm{k}$ at a point of homogeneous coordinates ${ }^{k} \boldsymbol{m}_{\mathrm{o}}^{\mathrm{X}}=\left[\begin{array}{lll}{ }^{\mathrm{k}} x_{\mathrm{o}}^{\mathrm{X}} & { }^{\mathrm{k}} y_{\mathrm{o}}^{\mathrm{X}} & 1\end{array}\right]^{\mathrm{T}}$. The corresponding coordinates in pixels will be denoted as ${ }^{\mathrm{k}} \boldsymbol{f}_{\mathrm{o}}^{\mathrm{X}}=\left[\begin{array}{lll}{ }^{\mathrm{k}} f_{\mathrm{xo}}^{\mathrm{X}} & { }^{\mathrm{k}} f_{\mathrm{yo}}^{\mathrm{X}} & 1\end{array}\right]^{\mathrm{T}}=\mathbf{A} \cdot{ }^{k} \boldsymbol{m}_{\mathrm{o}}^{\mathrm{x}}$ where $\mathbf{A}$ is a non-singular matrix which contains the intrinsic parameters of the camera being used:

$$
\mathbf{A}=\left[\begin{array}{ccc}
\mathrm{f} \cdot p_{\mathrm{u}} & -\mathrm{f} \cdot p_{\mathrm{u}} \cdot \cot (\theta) & u_{0} \\
0 & \mathrm{f} \cdot p_{\mathrm{v}} / \operatorname{sen}(\theta) & v_{0} \\
0 & 0 & 1
\end{array}\right]
$$

where $u_{0}$ and $v_{0}$ are the pixel coordinates of the principal point, $\mathrm{f}$ is the focal length, $p_{\mathrm{u}}$ and $p_{\mathrm{v}}$ are the magnifications in the $u$ and $v$ directions respectively, and $\theta$ is the angle between these axes.

Considering that a total of $\mathrm{M}$ points are obtained from the object $\mathrm{O}$, the discrete trajectory in the image space is also composed of $\mathrm{M}$ trajectories, $\boldsymbol{C}_{\mathrm{i}} / \mathrm{i} \in 1 \ldots \mathrm{M}$ (one for each feature extracted from the image that corresponds to the $M$ points). As such, the values of each of the $M$ trajectories in the image, at instants $\mathrm{k}$ are $\boldsymbol{C}_{\mathrm{i}}=\left\{{ }^{\mathrm{k}} \boldsymbol{f}_{\mathrm{i}} / \mathrm{k} \in 1 \ldots \mathrm{N}\right\}$. If the set of M features is considered as a desired configuration in the image, the set $\mathrm{S}$ is obtained, such that $\mathrm{S}=\left\{{ }^{\mathrm{k}} \mathbf{s} / \mathrm{k} \in 1 \ldots \mathrm{N}\right\}$, where ${ }^{\mathrm{k}} \mathrm{s}$ is the set of $\mathrm{M}$ points or features observed by the camera at instant $\mathrm{k}$, ${ }^{\mathrm{k}} \mathbf{s}=\left\{{ }^{\mathrm{k}} \boldsymbol{f}_{\mathrm{i}} / \mathrm{i} \in 1 \ldots \mathrm{M}\right\}$. Each sample, ${ }^{\mathrm{k}} \mathrm{s}$, is generated from each position ${ }^{\mathrm{k}} \boldsymbol{p}_{\mathrm{o}}^{\mathrm{x}}$. These positions are obtained considering that the time between two consecutive samples is constant, so that $\Delta \Delta^{\mathrm{k}+1} t={ }^{\mathrm{k}+1} t-{ }^{\mathrm{k}} t=T$ where $T$ is the video rate. The desired trajectory to be tracked in the image is obtained using a natural cubic B-spline:

$$
\mathbf{s}_{\mathrm{d}}(t)={ }^{\mathrm{k}} \mathbf{A} t^{3}+{ }^{\mathrm{k}} \mathbf{B} t^{2}+{ }^{\mathrm{k}} \mathbf{C} t+{ }^{\mathrm{k}} \mathbf{D}
$$

where ${ }^{\mathrm{k}} \mathbf{A},{ }^{\mathrm{k}} \mathbf{B},{ }^{\mathrm{k}} \mathbf{C},{ }^{\mathrm{k}} \mathbf{D}$ are obtained from the samples in the image space at the given instants.

To perform the tracking of the desired trajectory in the image space an image-based control scheme to regulate to 0 the following vision-based task function is used [5]:

$$
\boldsymbol{e}=\hat{\mathbf{J}}_{\mathrm{f}}^{+} \cdot\left(\mathbf{s}-\mathbf{s}_{\mathrm{d}}(t)\right)
$$

where $\mathbf{s}$ are the extracted features from the image and $\hat{\mathbf{J}}_{\mathrm{f}}^{+}$is an estimation of the pseudoinverse of the interaction matrix [2]. To carry out the tracking of the trajectory, the following velocity must be applied to the robot (with respect to the coordinate frame located in the eye-in-hand camera):

$$
\boldsymbol{v}^{\mathrm{C}}=-\mathrm{k} \cdot \boldsymbol{e}+\hat{\mathbf{J}}_{\mathrm{f}}^{+} \cdot \frac{\partial \mathbf{s}_{\mathrm{d}}(t)}{\partial t}
$$

where $\mathrm{k}>0$ is the gain of the proportional controller.

To assure that a given task in which it is required an interaction with the setting is correctly developed, the system must carry out a variation of the trajectory in the image, depending on the spatial restrictions imposed by the interaction forces. Therefore, given a collision with the setting and having recognized the normal vector of the contact surface, the transformation $\mathbf{T}$ that the camera must undergo to fulfil the spatial restrictions, is determined. This transformation is calculated so that it represents the nearest direction to the one obtained from the image-based control system, and which is contained in the plane of the surface. Thus, we guarantee that the visual information will be coherent with the sensorial information obtained from the force sensor. 
To do so, considering $\boldsymbol{f}$ to be the position of a given feature extracted by the camera at a given instant, and $\left[\mathbf{R}_{\mathrm{i}} \mathbf{t}_{\mathrm{i}}\right]$ a sampling of the transformation $\mathbf{T}$ that the camera undergoes during the tracking of the recognized surface, the feature $f_{\mathrm{i}}^{\prime}$ extracted in each one of these positions will be:

$$
\boldsymbol{f}_{\mathrm{i}}^{\prime}=\mathbf{A} \cdot \mathbf{R}_{\mathrm{i}} \cdot \mathbf{A}^{-1} \cdot \boldsymbol{f}+\mathbf{A} \cdot \mathbf{t}_{\mathrm{i}} / z
$$

where $\mathbf{A}$ is the intrinsic parameter matrix and $z$ is the distance between the camera and the object from which the features are extracted.

From the sampling of the desired trajectory in the image, $f_{\mathrm{i}}^{\prime}$, a spline interpolator is applied to obtain the desired trajectory in the image.

Once the coherence between both sensorial systems is guaranteed the system can use the visual and the force information coordinately to track a disassembly trajectory. The final control action, $\boldsymbol{v}^{\mathrm{C}}$, will be a weighted sum obtained from the visual servoing system, $\boldsymbol{v}_{\mathrm{M}}^{\mathrm{C}}$, and from the force sensor, $\boldsymbol{v}_{\mathrm{F}}^{\mathrm{C}}=\lambda_{\mathrm{F}} \cdot\left(\boldsymbol{F}-\boldsymbol{F}_{\mathrm{d}}\right) / k$ (where $F$ and $F_{\mathrm{d}}$ are the obtained and the desired interaction forces, $k$ is the tool stiffness, and $\lambda_{\mathrm{F}}$ is the proportional control gain for the force controller), so that $\boldsymbol{v}^{\mathrm{C}}=p_{\mathrm{v}} \cdot \boldsymbol{v}_{\mathrm{M}}^{\mathrm{C}}+p_{\mathrm{F}} \cdot \boldsymbol{v}_{\mathrm{F}}^{\mathrm{C}}$.

\section{RESULTS}

The proposed system is tested for the removal of a screw from a rail. First of all, the hierarchical model of the product, composed by the rail and the screw is modeled. With this model the global planner adjusts the database model to the real one, using an external stereo rig to perform this task, as it has been explained in Section 2. The hierarchical model is used by the sequence planner to determine the components to be separated to get accessibility to the desired one, the screw. In this case the component is directly accessible, due to the characteristics of the product that we are working on. Once, the component to be separated has been chosen, the trajectory to be performed has to be computed using the movement generator. In this case the trajectory obtained is to move the screw in a perpendicular direction to the rail and oriented to the top surface of the rail. At this point we should mention that since we are not interested in image processing issues in this paper, the tracked target is composed of four grey marks which will be the extracted features during the tracking (see Figure 2) and are adequate to control the task [2].

Considering this path to be followed by the screw, the robot detects a collision between the rail and the screw when the real task is been performed. To correct this situation a simultaneous control of the position and of the interaction of the robot has to be performed. To carry out this task, the experimental setup shown in Figure 2 is used.

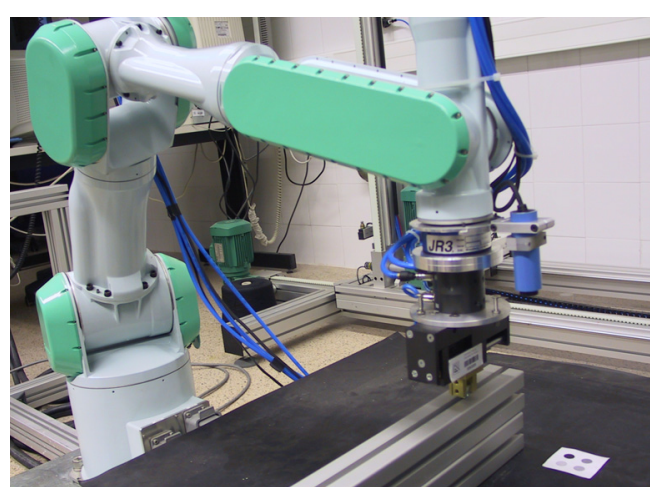

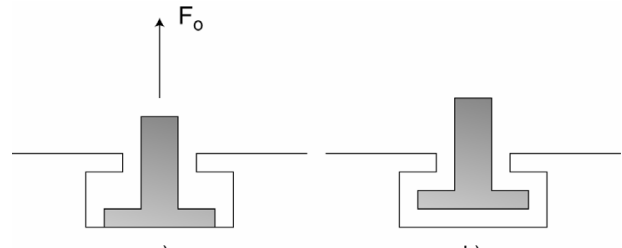

a)

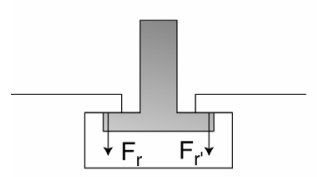

c) b)

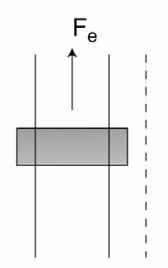

d)

Figure 2. Removal of the screw. 
Figure 2 shows the different phases required for the disassembly. The removal direction obtained by the movement generator is $\mathrm{F}_{\mathrm{o}}$, that can be used to move the screw from the initial position to the top surface of the rail. When the screw collides with this surface, the forces $F_{r}$ and $F_{r}$, appear in the opposite direction of $F_{o}$ (see Figure 2.c), these forces must be avoided for removing the screw. To achieve the correct removal of the component, the new direction $F_{e}$ is generated for removing the component from the rail. This new direction can be seen in a perpendicular view of the rail in Figure 2.d.

Obviously, when the disassembly is been done in the direction indicated in Figure 2.a, the head of the screw will eventually collide with the rail, so that, the disassembly will no longer be possible. This happens if the screw is not perfectly modeled, i.e., it is modeled as a cylinder but the head of the screw is not considered.

The global planner generates the model of the product fusing the information from the database and the stereo rig. Therefore, the geometric model obtained is not the perfect model of the component. This simple model also allows an easy way of performing the generation of the trajectory computed for the disassembly of the component by the movement generator.

In such a case, the situation shown in Section 3 arises. That is, there is a contradiction between the direction established by the vision system and the interaction information obtained from the force-sensor. In accordance with the algorithm presented in Section 3, and once the contact surfaces has been recognized, the next step will be to modify the trajectory in the image, using the information from the force-sensor, so that the new movement direction will be coherent with the interaction information obtained.

In this task, the screw in the rail shown in Figure 2 is to be removed. In this figure the pattern with four marks, which will be extracted by using the eye-in-hand camera system during the tracking, is shown. Therefore, the desired trajectory in the image will be generated by using these marks.

To carry out the removal of the screw, its head must maintain a constant force against one side of the rail during the disassembly ( $1 \mathrm{~N}$. in $\mathrm{x}$ direction), and also against the upper part $(5 \mathrm{~N}$. in $\mathrm{z}$ direction). Once the robot has grasped the screw and the contact surface is recognized, the desired trajectory in the image is generated to carry out the disassembly process. This trajectory is used by the image-based control system (see Equation 4) to develop the disassembly trajectory.

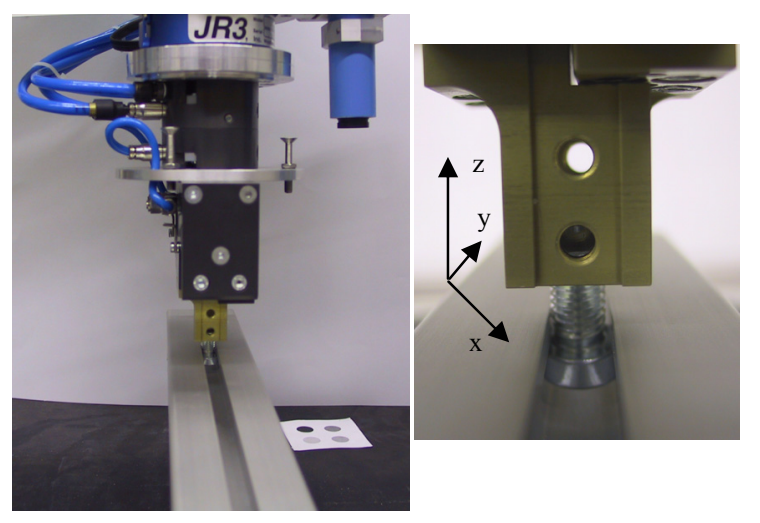

Force in N

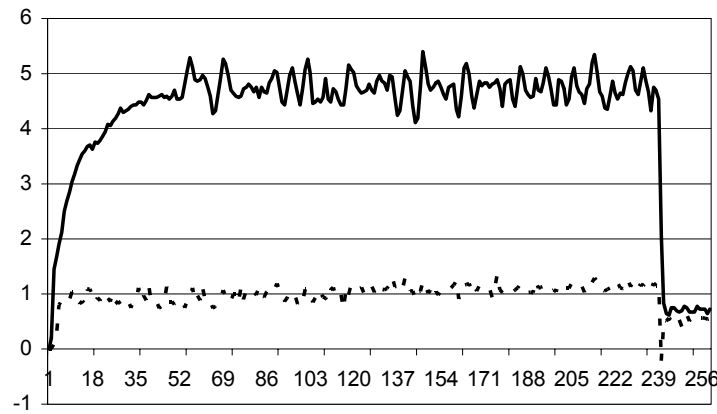

Figure 3. Evolution of the generated forces during the disassembly process.

In Figure 3, the evolution of the forces obtained in the joint use of the force and visual control, is shown. In this figure, we can observe that once the forces are established at their desired levels, 
the displacement of the screw along of the rail is carried out. In the last phase of the graph, the forces are quickly annulled and, therefore, the disassembly process is finished.

\section{CONCLUSIONS}

The different modules of a generic flexible disassembly system have been described in this paper. This system allows the generation of trajectories to be performed by a robot arm and a multi-sensorial system to track these trajectories is also shown.

The use of a multi-sensorial system for the generation of disassembly trajectories increases the performance of the system because this allows it to take unknown situations into consideration. The fusion of force and visual data is a good approach for removal operations due to these sensors allow the system to react according to the real product that is been disassembled, modifying the trajectory based on the model of the product.

The force-sensor gives information about the contact between the component to be removed and the rest of the product, which is useful when occlusions of the component to be removed appear during the disassembly process.

We are now working in improving the geometric model according the sensorial information acquired during the disassembly process. This aspect will allows the system to generate better trajectories as the system is used.

\section{ACKNOWLEDGEMENTS}

This work was funded by the Spanish MCYT project "Diseño, implementación y experimentación de escenarios de manipulación inteligentes para aplicaciones de ensamblado y desensamblado automático", by the project GV05/007: "Diseño y experimentación de estrategias de control visual-fuerza para sistemas flexibles de manipulación" and by the Spanish G.V. project "Desensamblado automático cooperativo para el reciclado de productos" (GV05/003).

\section{REFERENCES}

[1] J. E.,Boon, J. A. Isaacs, S. M. Gupta, S.M. "Economic sensitivity for end of life planning and processing of personal computers". Journal of Electronic Manufacturing, Vol. 11, No. 1, 2002. pp 81-93.

[2] F. Chaumette, "Potential problems of stability and convergence in image-based and positionbased visual servoing", The Confluence of Vision and Control, D. Kriegman, G . Hager, A. Morse, Eds., LNCIS Series, Springer Verlag. Vol. 237, 1998, pp. 66-78.

[3] P. Gil, S. T. Puente, F. Torres, J. Pomares, F. A. Candelas, "Data fusion from multiples cameras for automatic disassembly". IFAC Workshop on Intelligent Assembly and Disassembly - IAD'2001. 2001.

[4] A. J. D. Lambert. "Disassembly sequencing: a survey". International Journal of Production Research, Vol. 41, No. 16, 2003, pp. 3721-3759.

[5] Y. Mezouar, F. Chaumette. "Path Planning For Robust Image-based Control". IEEE Transactions on Robotics and Automation, Vol. 18, No. 4, 2002, pp. 534-549.

[6] L. K. Moyer, S. M. Gupta. "Environmental concerns and recycling/disassembly efforts in the electronics industry". Journal of Electronics Manufacturing, Vol. 7, No. 1, 1997, pp. 1-22.

[7] J. Pomares, F. Torres, S. T. Puente " Disassembly movements for geometrical objects through heuristic methods". SPIE: Environmentally Conscious Manufacturing II, Vol. 4569, Boston, USA, 2001, pp. 71-80.

[8] S. T. Puente, F. Torres, R. Aracil, R. "Non-Destructive Disasembly Robot Cell for Demanufacturing Automation". IFAC Workshop on Intelligent Assembly and Disassembly IAD'2003. Bucharest, (Romania). 2001, pp. 126-131.

[9] F. Torres, S. T. Puente, R. Aracil. "Disassembly planning based on precedence relations among assemblies". International Journal of Advanced Manufacturing Technology, Vol. 31, N. 5, 2003, pp. 317-327. 